\title{
Combining simulations and solution experiments as a paradigm for RNA force field refinement
}

\author{
Andrea Cesari, Alejandro Gil-Ley, and Giovanni Bussi* \\ Scuola Internazionale Superiore di Studi Avanzati (SISSA), via Bonomea 265, 34136, Trieste, \\ Italy \\ E-mail: bussi@sissa.it
}

\begin{abstract}
Recent computational efforts have shown that the current potential energy models used in molecular dynamics are not accurate enough to describe the conformational ensemble of RNA oligomers and suggest that molecular dynamics should be complemented with experimental data. We here propose a scheme based on the maximum entropy principle to combine simulations with bulk experiments. In the proposed scheme the noise arising from both the measurements and the forward models used to back calculate the experimental observables is explicitly taken into account. The method is tested on RNA nucleosides and is then used to construct chemically consistent corrections to the Amber RNA force field that allow a large set of experimental data on nucleosides and dinucleosides to be correctly reproduced. The transferability of these corrections is assessed against independent data on tetranucleotides and displays a previously unreported agreement with experiments. This procedure can be applied to enforce multiple experimental data on multiple systems in a self-consistent framework thus suggesting a new paradigm for force field refinement.
\end{abstract}

\section{Introduction}

Molecular dynamics (MD) simulations in explicit solvent have been successfully applied to RNA systems in order to describe dynamics around the native structure as well as small conformational changes. $^{1-9}$ In the case of RNA folding, a few partly successful atomistic simulations have been reported. ${ }^{10-13}$ However, recent extensive simulations of unstructured oligonucleotides for which converged sampling is affordable have unambiguously shown that current force-field parameters are not accurate enough to reproduce solution experiments. ${ }^{14-18}$ These results prompt for a new effort in RNA force field refinement and suggest that MD should be complemented with experimental data, when available. An important point here is the realization that many experimental techniques intrinsically provide values that are averaged both over time and over a large ensemble of replicates of the same molecule. In this respect, many ways to enforce averages that match experiments have been proposed in the past ${ }^{19-24}$. The maximum entropy (MaxEnt) principle allows ensemble averages to be constrained and is a natural framework to achieve this goal ${ }^{25,26}$. For instance, White and Voth implemented the MaxEnt procedure in their experimentally directed simulation method $(E D S)^{27}$. On top of this, recent works have underlined the importance of taking into account experimental errors when using these constraints ${ }^{28-30}$. This latter step is often done in a Bayesian framework, which is not designed to provide a single optimal model but to sample the posterior distribution thus generating ensembles of solutions compatible with the constraints.

In this work, we propose a novel method to enforce experimental data in MD simulations. The method is an extension of the MaxEnt framework and includes auxiliary variables to model exper- 
imental errors. Being based on MaxEnt, this procedure allows a modified potential energy function to be estimated on the fly. This method can be straightforwardly combined with replica-exchange methods so as to enhance sampling and improve the convergence of the modified potential. We apply the method to the difficult task of designing a RNA force field that matches NMR data from ${ }^{3} J$ scalar couplings. The force field is restrained to have a consistent functional form where chemically equivalent atoms are subject to equivalent terms. The method is trained on a set of nucleosides and dinucleoside monophosphates where it can reproduce solution experiments with a previously unreported accuracy. Finally, the obtained corrections are combined with a recently proposed correction on the RNA backbone ${ }^{17}$ and validated using converged ensembles of tetranucleotides, where they significantly improve the agreement with independent solution experiments, including ${ }^{3} J$ scalar couplings and nuclear-Overhauser-effect (NOE) data. The success of the procedure suggests a new paradigm for force field refinement.

\section{Method}

\subsection{MaxEnt approach}

The rationale behind the maximum entropy (MaxEnt) approach, as firstly proposed by Jaynes ${ }^{31}$, is to find a normalized probability distribution $P(x)$ which satisfies a set of constraints and minimizes the Kullback-Leibler divergence ${ }^{32}$ from a reference probability $P_{0}(x)$, defined as:

$$
D_{K L}\left(P \| P_{0}\right)=\int d x P(x) \ln \frac{P(x)}{P_{0}(x)},
$$

Here $P_{0}(x)$ expresses our prior information on the system and should be interpreted as the canonical distribution associated with the initial, unrefined potential energy function. Minimizing $D_{K L}$ is equivalent to maximizing the relative entropy between $P$ and $P_{0}$ and, by construction, provides an ensemble that introduces the minimum possible amount of information with respect to the prior knowledge. The ensemble averages of a set of $M$ observables $f_{i}(x) i=1, \ldots, M$, in the refined ensemble $P(x)$, are equal to $\left\langle f_{i}\right\rangle=\int d x P(x) f_{i}(x)$ and should be constrained to their experimental values $f_{\text {exp }, i}$ :

$$
f_{\text {exp }, i}=\int d x P(x) f_{i}(x) .
$$

Here $f_{i}(x)$ is a function of the atomic coordinates of the system and depends on the nature of the performed experiment. For example, three-bond scalar couplings ${ }^{3} J(\theta)$ are related to the dihedral angles formed by the 4 involved atoms according to the Karplus relations ${ }^{33}: f(\theta)={ }^{3} J(\theta)=$ $A \cos ^{2} \theta+B \cos \theta+C$. Minimizing the functional in equation (1) using the method of Lagrangian multipliers leads to:

$$
P(x)=\frac{P_{0}(x) e^{-\sum_{i=1}^{M} \lambda_{i} f_{i}(x)}}{\int d x P_{0}(x) e^{-\sum_{i=1}^{M} \lambda_{i} f_{i}(x)}} .
$$

In the rest of the paper we will refer to this distribution as posterior distribution. Here $\lambda$ is an array of Lagrangian multipliers that should be determined in a self-consistent procedure. This corresponds to a refined potential equal to:

$$
U(x)=U_{0}(x)+k_{B} T \sum_{i=1}^{M} \lambda_{i} f_{i}(x)
$$

It has been shown by Voth et al. ${ }^{34}$ that such functional form of the biasing potential will always reduce the relative entropy with respect to an ideal ensemble, in which all observable are in agreement with experiments.

To determine the Lagrangian multipliers it is convenient ${ }^{26,35}$ to define a function $\Gamma(\lambda)=$ $\ln \int d x e^{-\beta U(x)}+\sum_{i=1}^{M} \lambda_{i} f_{\text {exp }, i}$. The gradient of $\Gamma$ is

$$
\frac{\partial \Gamma}{\partial \lambda_{i}}=f_{\text {exp }, i}-\left\langle f_{i}(x)\right\rangle \text {. }
$$

Since for uncorrelated observables the Hessian of $\Gamma$ is positive definite, a set of Lagrangian multipliers $\left\{\lambda^{*}\right\}$ satisfying the constraints in equation (2) can be found by minimizing $\Gamma$. In the case of correlated observables the function $\Gamma$ becomes positive semidefinite changing its character from strictly convex to convex. This implies that there could exist multiple choices of $\left\{\lambda^{*}\right\}$ satisfying the constraints. It can be shown however that if the constraints are satisfied by different set of $\left\{\lambda^{*}\right\}$, they all can be considered as acceptable solutions 
to the problem (see Supporting Information subsection 1.1).

The optimal values for $\lambda$ can be computed on the fly during a MD simulation using a stochastic procedure. Similarly to Ref. ${ }^{27}$, we use a stochastic gradient descent method where $\lambda$ is updated according to

$$
\dot{\lambda}_{i}(t)=-\eta_{i}(t)\left(f_{\exp , i}-f_{i}(x(t))\right)
$$

Here $\eta$ is a suitable learning rate which we chose from the class type "search then converge" as $\eta(t)=\frac{k_{i}}{1+t / \tau_{i}}$ following $\operatorname{Ref}^{36}$. Here $k_{i}$ represents the initial learning rate and $\tau_{i}$ represents its damping time. In this manner, the learning rate is large at the beginning of the simulation and decreases proportionally to $1 / t$ for large simulation times. Interestingly, a $1 / t$ schedule for the learning rate has been found to be optimal also in the context of enhanced sampling methods ${ }^{37}$. The simulation can be stopped when Lagrangian multipliers are converged. If required, the simulation can be continued using a static correcting potential. The best estimate of the values of $\lambda$ satisfying the experimental constraints is given by the time average $\lambda_{i}^{*}$ of the Lagrangian multipliers over an appropriate time window $\left[t_{\min }, t_{\max }\right]$. In the rest of the paper, we call "learning phase" the initial part of the simulation $\left(t<t_{\max }\right)$, "averaging phase" the portion of the learning phase where $\lambda$ is averaged $\left(t_{\text {min }}<t<t_{\text {max }}\right)$, and "production phase" the later part of the simulation $\left(t>t_{\max }\right)$, where $\lambda$ is kept equal to the computed average. It should be mentioned that the procedure used here is related to a recently introduced a variationally enhanced sampling approach ${ }^{38}$ where a similar stochastic minimization is used to enforce target distributions during a MD simulation.

\subsection{Inclusion of experimental errors}

The procedure outlined above is appropriate when available experimental data can be assumed to be exact. However, in practical cases many sources of error can affect the data, including systematic and random errors on the data as well as errors arising from a sub-optimal parametrization for the functions $f_{i}(x)$. In our example, the latter case would correspond to a sub-optimal estimation of the parameters in the Karplus relations. Requiring a perfect agreement with experiments could lead to huge Lagrangian multipliers. We here extend the MaxEnt formalism so as to explicitly account for all the above mentioned errors. Namely, we consider an extended system where an additional variables $\varepsilon_{i}$ is introduced to take into account the deviation between the experimental data $f_{\text {exp }, i}$ and the ensemble average $\left\langle f_{i}\right\rangle$. The enforced constraints are thus

$$
\left\langle\left(f_{i}(x)+\varepsilon_{i}\right)\right\rangle=f_{\text {exp }, i}
$$

A priori, the variables $\varepsilon_{i}$ are considered to be independent of the atomic coordinates $x$, and with a prior probability distribution $P_{0}(\varepsilon)$. To keep the notation simple, we denote with the same letter both the prior on the atomic coordinates $P_{0}(x)$ and the prior on the deviation $P_{0}(\varepsilon)$, as these two functions can be easily distinguished by their argument. The prior $P_{0}(\varepsilon)$ can be used to model the experimental errors. In the simplest case, one can assume it to be a Gaussian function with a given standard deviation $\sigma_{0}, P_{0}(\varepsilon)=\frac{e^{-\frac{\varepsilon^{2}}{2 \sigma_{0}^{2}}}}{\sqrt{2 \pi} \sigma_{0}}$. Here a single experimental value has been assumed $(M=1)$, but equations are straightforwardly generalized to multiple data. Different values of $\sigma_{0}$ can be chosen for different types of experiment. In more general cases, if $\sigma_{0}$ is difficult to choose, one can consider it as an additional variable with an appropriate prior distribution $P_{0}\left(\sigma_{0}\right)$. The resulting prior for $P_{0}(\varepsilon)$ can thus be obtained by marginalization.

Once the prior $P_{0}(\varepsilon)$ has been defined, one should enforce the constraint in equation (7). This is done by applying the MaxEnt procedure on the extended system defined by the coordinates $\boldsymbol{x}$ and the auxiliary variables $\varepsilon_{i}$. The resulting posterior distribution is

$$
P(x, \varepsilon)=\frac{P_{0}(x) P_{0}(\varepsilon) e^{-\sum_{i} \lambda_{i}\left(f_{i}(x)+\varepsilon_{i}\right)}}{\int d x d \varepsilon P_{0}(x) P_{0}(\varepsilon) e^{-\sum_{i} \lambda_{i}\left(f_{i}(x)+\varepsilon_{i}\right)}}
$$

As a consequence of this exponential form, $\boldsymbol{x}$ and $\varepsilon$ are independent also in the posterior distribution and hence can be factorized. The enforced constraint can thus be written as

$$
\left\langle f_{i}(x)\right\rangle+\left\langle\varepsilon_{i}\right\rangle=f_{\text {exp }, i}
$$


The average value of $\varepsilon$ depends only on the value of $\lambda$ and can be analytically computed without explicitly sampling $\varepsilon$ as:

$$
\xi_{i}(\lambda) \equiv\left\langle\varepsilon_{i}\right\rangle=\frac{\int d \varepsilon P_{0}(\varepsilon) e^{-\sum_{j} \lambda_{j} \varepsilon_{j}} \varepsilon_{i}}{\int d \varepsilon P_{0}(\varepsilon) e^{-\sum_{j} \lambda_{j} \varepsilon_{j}}}
$$

where $\xi_{i}(\lambda)$ is defined as the average value of $\varepsilon$ in the posterior distribution. Once the functional form of $\xi_{i}(\lambda)$ is known, one should enforce the average values obtained from the MD simulation to be equal to $f_{\text {exp }, i}-\xi_{i}(\lambda)$. By applying the stochastic minimization procedure described above to the extended system one obtains the following update rule for $\lambda$

$$
\dot{\lambda}_{i}(t)=-\eta(t)\left(f_{\text {exp }, i}-\xi_{i}(\lambda)-f_{i}(x(t))\right)
$$

This equation is the most important of this paper and completely describes the algorithm that we use to restrain MD with noisy experimental data. The treatment of the error is fully enclosed in the functional form of $\xi_{i}(\lambda)$. The equations of motion are integrated using the algorithm reported in Supporting Information (subsection 1.2), where it is also explained how to include experiments providing only an upper or lower limit for a given observable. Since $\lambda$ changes during the simulation, the system is kept out of equilibrium. The work performed can be estimated (see Supporting Information equation 1.2) and used to compute the effective energy drift ${ }^{39}$.

In the simple case where $P_{0}(\varepsilon)$ is a Gaussian with standard deviation $\sigma$ the $\xi$ function is

$$
\xi_{i}(\lambda)=-\lambda_{i} \sigma^{2}
$$

Larger values of $\sigma$ would lead to smaller Lagrangian multipliers at the end of the stochastic minimization. The value of $\sigma$ thus tells us how much we trust in the original force field and is related to the value of $\theta$ introduced in $\operatorname{Ref}^{29}$. Non Gaussian prior distributions for $\varepsilon$ can be used to better tolerate outliers. The Laplace prior $P_{0}(\varepsilon) \propto$ $e^{-\sqrt{2} \frac{|\varepsilon|}{\sigma}}$ results in

$$
\xi_{i}(\lambda)=-\frac{\lambda_{i} \sigma^{2}}{1-\frac{\lambda^{2} \sigma^{2}}{2}}
$$

This function is similar to the one in equation (9) for small values of $\lambda$. However, when $\lambda$ approaches the limiting value of $\frac{\sqrt{2}}{\sigma}, \xi$ diverges and arbitrarily large discrepancies with the experimental data are accepted. This procedure forces $\lambda$ to be in the domain $\left[-\frac{\sqrt{2}}{\sigma},+\frac{\sqrt{2}}{\sigma}\right]$, and thus intrinsically limits the strength of the corrections to the original force field. We notice that these boundaries ensure that the posterior $P(\varepsilon) \propto e^{-\sqrt{2} \frac{|\varepsilon|}{\sigma}} e^{-\lambda \varepsilon}$ can be normalized.

A general class of priors $P_{0}\left(\sigma_{0}\right)$ is presented in Supporting Information (subsection 1.3) that comprises the above mentioned Gaussian and Laplace priors $P_{0}(\varepsilon)$ as special cases. We notice that, although in this examples $\xi_{i}$ only depends on $\lambda_{i}$, in principle any element of $\xi$ could depend on the value of any of the Lagrangian multipliers. This could happen when errors on different experimental data are considered as correlated (see Supporting Information subsection 1.4).

\subsection{Self-consistent force-field refine- ment}

Once the set of $\left\{\lambda^{*}\right\}$ satisfying equation (7) are determined, the potential energy used in the MaxEnt framework (equation (4)) is equivalent to the original force field plus a correction linear in the experimental observables. Although these corrections have been fitted to match experiments on one specific system, one could try to transfer them to a system different from the original one. This is particularly appealing in the case of ${ }^{3} J$ couplings, where the functional form of the correction is comparable to the standard torsional terms that are present in biomolecular force fields. We propose here to transfer the parameters directly during the learning phase. If experimental data are available for a number of similar systems, one should simulate all the systems in parallel. Each of the simulated systems will be affected both by the corrections arising from the experiments performed on the same system and by some of the correcting potentials determined by the other simulated systems. This procedure allows to fit force-field corrections in a self-consistent procedure that restrains some of the terms to be equivalent to each other. In the case of corrections derived by ${ }^{3} \mathrm{~J}$ couplings, it is possible to enforce the same correction 
on dihedrals that are chemically equivalent to each other. For instance, the torsional potential of a $\chi$ angle in an adenine is expected to be the same irrespectively of its position in the sequence. A detailed description of the procedure can be found in Supporting Information (subsection 1.5). The systems considered are: A, C, ApA, ApC, CpA, and $\mathrm{CpC}$. Experimental data for the dinucleoside monophosphates were taken from Refs. ${ }^{40-42}$

\subsection{Molecular dynamics}

We performed molecular dynamics on RNA nucleosides (A and $\mathrm{C}$ ) and dinucleoside monophosphates (ApA, ApC, CpA, and $\mathrm{CpC}$ ). Molecular dynamics simulations were performed using the GROMACS software package ${ }^{43}$ in combination with a modified version of the PLUMED plugin ${ }^{44}$. RNA, explicit water, and ions were modeled using the most recent parametrizations within the Amber force field ${ }^{1,45-49}$. Parameters are available at http://github.com/srnas/ff. Bonds were constrained using the LINCS algorithm ${ }^{50}$, allowing for a timestep of $2 \mathrm{fs}$. The particle-mesh Ewald algorithm ${ }^{51}$ was used for long-range electrostatic interactions with a cut-off distance of 1 $\mathrm{nm}$. Simulations were performed at temperature $\mathrm{T}=300 \mathrm{~K}$ and pressure $\mathrm{P}=1 \mathrm{bar}^{52,53}$. To allow for a fast convergence of the simulated ensembles, sampling was enhanced using replica-exchange with collective-variable tempering (RECT) ${ }^{54}$ on selected collective variables. For the nucleosides we biased the torsional angles $\chi, \gamma$, and the puckering variables $Z_{x}$ and $Z_{y}{ }^{55}$. For the dinucleoside monophosphates we additionally included torsional angles $\alpha, \beta, \varepsilon$, and $\zeta$ as well as the distance between the two nucleobases. Four replicas were used for each system, with bias factors ranging from 1 to 5 both for the nucleosides and dinucleoside monophosphates.

\subsection{MaxEnt algorithm parameters}

For the nucleosides we performed $200 n s$ MD per replica using the first $100 n s$ as learning phase. Lagrangian multipliers were averaged from $t_{\text {min }}=50 \mathrm{~ns}$ to $t_{\max }=100 \mathrm{~ns}$ and these averages were used in the production phase for the last $100 n s$. For the dinucleoside monophosphates we performed $600 n s$ using first $300 n s$ as learning phase and averaging Lagrangian multipliers between $t_{\text {min }}=150 \mathrm{~ns}$ and $t_{\max }=300 \mathrm{~ns}$.

The parameters for the learning phase were chosen as $k=0.001 \mathrm{~Hz}^{-2} \mathrm{ps}^{-1}, \tau=3 \mathrm{ps}, \sigma=$ 2.0 $\mathrm{Hz}$ for both the nucleosides and the dinucleoside monophosphates. In both cases a Laplace prior for the error was used. The biased replicas were simulated using Lagrangian multipliers estimated on the fly from the reference replica, so as to maximize the acceptance rate for the replica-exchange procedure. To implement the self-consistent force-field fitting described above, we simultaneously simulated six systems (A, C, $\mathrm{ApA}, \mathrm{ApC}, \mathrm{CpA}$, and $\mathrm{CpC})$. The replica exchange framework of GROMACS was used, disallowing unphysical exchanges between replicas simulating different systems. Each system was simulating with 4 RECT replicas, resulting in a total of 24 replicas. Lagrangian multipliers were adjusted to fit experimental data available for each of the systems and transmitted on the fly to the other replicas so as to be applied on all the equivalent dihedrals. Input files are provided in Supporting Information (figure S5-S9). The modifications to PLUMED required to perform this simulations are available on request and will be included in the next PLUMED release.

\section{Results}

In the following we show a number of applications of the discussed method. First we enforce data from solution experiments on RNA nucleosides, showing that the procedure can be used to construct an ensemble compatible with experiments. Then, the self-consistent procedure is used to fit force-field corrections for nucleosides and dinucleosides composed of adenine and cytosine. The obtained corrections are validated on the difficult case of RNA tetranucleotides. A model onedimensional system which is designed to highlight the features of the different models for including the experimental errors is shown in Supporting Information (see subsection 2.1) 


\subsection{Enforcing ${ }^{3} J$ coupling on a nucle- oside}

We show here an application of the introduced procedure to an RNA nucleoside. We here discuss results for adenosine only. Results for other nucleosides (uridine, cytidine, and guanosine) are similar and are summarized in Supporting Information (table $\mathrm{S} 1$ ). For this system, $M=7$ experimental ${ }^{3} J$ scalar couplings are available ${ }^{56}$, involving dihedral angles both on the backbone and on the nucleobase (see figure 1a).

a)

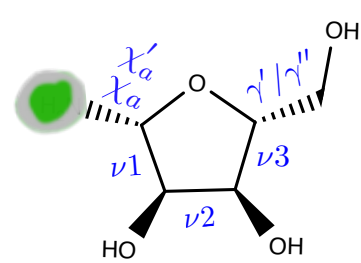

Adenosine

A

b)

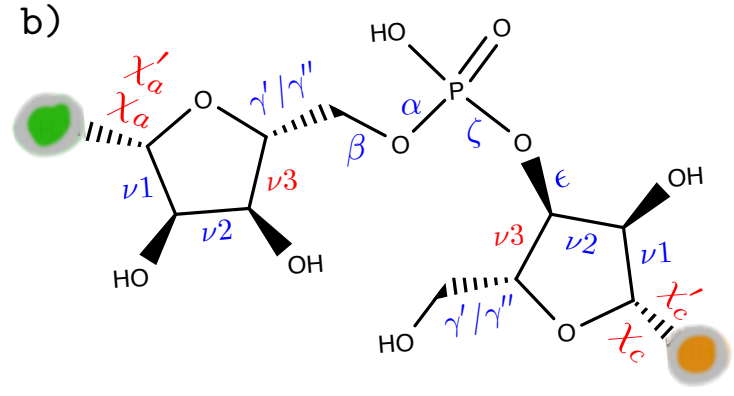

$$
\begin{gathered}
\text { Adenylyl-( } \left.3^{\prime} \rightarrow 5^{\prime}\right) \text {-cytidine } \\
\text { ApC }
\end{gathered}
$$

Figure 1: Torsional angles associated to the available experimental ${ }^{3} J$ scalar couplings for the Adenosine (panel a) and the ApC dinucleoside (panel b). Atoms associated to each torsion are: $v_{1}=\mathrm{H} 1^{\prime}-\mathrm{C}^{\prime}-\mathrm{C} 2$ '- $\mathrm{H} 2$ ', $v_{2}=\mathrm{H}^{\prime}-\mathrm{C}^{\prime}-\mathrm{C}^{\prime}-\mathrm{H}^{\prime}, v_{3}=\mathrm{H}^{\prime}-\mathrm{C} 3^{\prime}-\mathrm{C} 4^{\prime}-\mathrm{H}^{\prime}, \gamma^{\prime}=\mathrm{H} 4^{\prime}-$ C4'-C5'-H5', $\gamma^{\prime \prime}=\mathrm{H}^{\prime}$ '-C4'-C5'-H5', $\varepsilon_{1}=\mathrm{C} 4^{\prime}-\mathrm{C} 3^{\prime}-03^{\prime}-\mathrm{P}$, $\zeta_{1}=\mathrm{C}^{\prime}-03^{\prime}-\mathrm{P}-05^{\prime}, \alpha_{2}=03^{\prime}-\mathrm{P}-05^{\prime}-\mathrm{C}^{\prime}, \beta_{2}=\mathrm{P}-05^{\prime}-\mathrm{C}^{\prime}$ 'C4', $\chi_{A}=04^{\prime}-\mathrm{C} 1$ '-N9-C4, $\chi_{A}^{\prime}=\mathrm{H} 1^{\prime}-\mathrm{C} 1^{\prime}-\mathrm{N} 9-\mathrm{C} 8+60^{\circ}$, $\chi_{C}=\mathrm{O}^{\prime}-\mathrm{C} 1{ }^{\prime} \mathrm{N} 1-\mathrm{C} 2, \chi_{C}^{\prime}=\mathrm{H} 1^{\prime}-\mathrm{C}^{\prime}{ }^{\prime}-\mathrm{N} 1-\mathrm{C} 6+60^{\circ}$

We assess the deviation between simulation and experiments by computing the RMSE of the back calculated data from their experimental values, defined as $\sqrt{\frac{1}{M} \sum_{i=1}^{M}\left({ }^{3} J_{i, \text { simulated }}-{ }^{3} J_{i, \text { exp }}\right)^{2}}$. We first computed the scalar couplings using the stan- dard Amber force field (see table 1). The RMSE in this case is $1.3 \mathrm{~Hz}$. This number is significantly larger than the expected experimental error. However, it is important to consider also errors in the parametrization of the Karplus equations. To this aim, we compared a set of commonly used parametrizations (see Supporting Information subsection 1.6) and computed their standard deviation on the trajectory corresponding to the $\mathrm{ApC}$ dinucleoside monophosphate (reported in the next section), which resulted in approximately $0.6 \mathrm{~Hz}$. This number is significantly smaller than the RMSE observed for the Amber force field. This test also indicates that enforcing an RMSE between simulation and experiment lower than 0.6 could lead to results dependent on the choice of the Karplus equation parameters.

Additionally, we estimated the ability of random conformations to reproduce the experimental ${ }^{3} \mathrm{~J}$ scalar couplings. To this aim, we computed the RMSE between simulation and experiments assuming a flat distribution on all the torsions used in the ${ }^{3} \mathrm{~J}$ coupling calculation. The torsions considered were the ones available for the ApC dinucleosides with the same set of Karplus parameters which was used to produce all the results in this work. The resulting RMSE is approximately 2.9 $\mathrm{Hz}$, indicating that random conformations do not reproduce experimental data with the accuracy of MD ensembles.

We then use our iterative procedure to determine the correcting potentials. Although we use a Laplace prior for the error, we notice that since the correcting potential has as many degrees of freedom as experimental data, one cannot expect to detect inconsistencies in the dataset. A crucial parameter in the fitting procedure is $\sigma$, which controls the width of the prior distribution for the deviation between experiment and theory, and encodes the confidence that we have in the force field. Results for $\sigma=2.0 \mathrm{~Hz}$ are shown in table 1. As it can be seen, the RMSE is greatly reduced compared to the original Amber force field. Lagrangian multipliers are shown in Supporting Information (table $\mathrm{S} 2$ ). We recall that the greater the value of $\sigma$ the higher the confidence in the force field and the lower the correcting MaxEnt potential. A plot of RMSE vs $\sigma$ is provided in Supporting Information (figure S3). We notice that in this case an ar- 

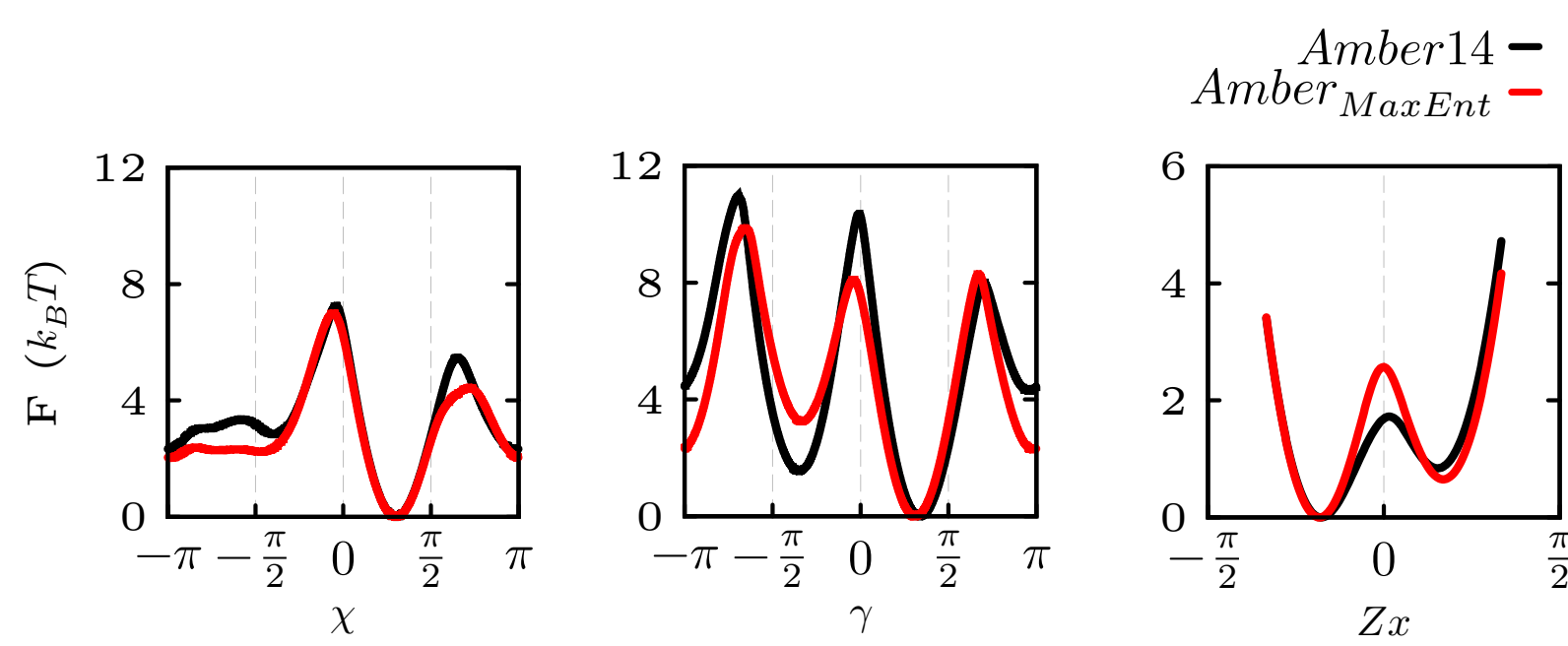

Figure 2: One-dimensional free-energy profiles for a representative group of the corrected dihedral angles obtained with Amber and with the refined Amber ${ }_{M a x E n t}$ force fields. $Z_{x}$ variable ${ }^{55}$ is related to sugar conformations $\mathrm{C}^{\prime}$ '-endo $\left(Z_{x}>0\right)$ and C2'-endo $\left(Z_{x}<0\right)$.

Table 1: ${ }^{3} J$ scalar coupling for the Adenosine nucleoside. Experimental values and back calculated values are shown, both using the Amber force field and the MaxEnt corrections. Angle $\chi^{\prime}$ for the Adenosine nucleoside is defined as the $H 1^{\prime}-C 1^{\prime}-N 9-C 8$ torsion along with a shift of $60^{\circ}$. Statistical errors on the values obtained from MD as well as on the calculated RMSE are less than $0.1 \mathrm{~Hz}$.

\begin{tabular}{|c|c|c|c|}
\cline { 2 - 4 } \multicolumn{1}{c|}{} & \multicolumn{3}{c|}{${ }^{3} J$ coupling $(\mathrm{Hz})$} \\
\hline torsion & Exp. $^{56}$ & Amber & Amber $_{\text {MaxEnt }}$ \\
\hline \hline$v_{1}$ & 6.0 & 8.5 & 6.9 \\
\hline$v_{2}$ & 5.0 & 5.1 & 5.1 \\
\hline$v_{3}$ & 3.4 & 3.5 & 4.2 \\
\hline$\gamma^{\prime}$ & 3.0 & 3.2 & 3.1 \\
\hline$\gamma^{\prime}$ & 3.4 & 1.5 & 2.6 \\
\hline$\chi$ & 3.6 & 4.7 & 4.1 \\
\hline$\chi^{\prime}$ & 3.9 & 3.6 & 3.5 \\
\hline \multirow{3}{*}{} & \multicolumn{3}{|c|}{$R M S E(\mathrm{~Hz})$} \\
\cline { 2 - 4 } & 0.0 & 1.3 & 0.6 \\
\cline { 2 - 4 } & \multicolumn{3}{c}{}
\end{tabular}

bitrary small RMSE can be obtained by choosing a negligible value of $\sigma$. It should be noticed that enforcing a RMSE smaller than the typical RMSE between different set of parameters in Karplus relations $(\approx 0.6 \mathrm{~Hz})$ is not meaningful. Moreover, this would introduce much larger corrections to the force field (see Supporting Information figure S4) that could lead to uncontrolled artifacts. For instance, in some of the simulations using $\sigma=0$ we obtained stereoisomerizations of the C2' atom of the sugar (data not shown). With the adopted value of $\sigma=2$ the effect of the corrections on the onedimensional free-energy profiles of the refined dihedral angles is $\leq 2 K_{b} T$. Free-energy profiles for a set of representative torsional angles are shown in figure 2 .

\subsection{Using ${ }^{3} J$-coupling for self-consistent force-field refinement}

We use the introduced procedure to perform a selfconsistent force-field refinement on a set of RNA nucleosides and dinucleoside monophosphates. In figure $1 \mathrm{~b}$ the $\mathrm{ApC}$ dinucleoside is shown and all the torsions considered in the refinement procedure are indicated. The obtained Lagrangian multipliers for each torsional angle are summarized in table 2. When fitting systems involving different nucleobases (e.g A and C), torsions around the glycosidic bond were considered as base dependent, together with the $v_{3}$ torsion, which we em- 
pirically observed to be the sugar torsion that is most correlated with the base/sugar relative orientation. Such torsions will feel a different correcting potential depending on whether they belong to and Adenosine or Cytosine. Base dependent torsions are highlighted in red in figure $1 \mathrm{~b}$. In case of a duplicated term in a single simulation (e.g., the $\chi$ angle in an adenine which appears twice in the ApA dinucleoside monophosphate), we do not enforce their individual values but the sum of the two scalar couplings to match the sum of the corresponding experimental values.

RMSEs for each system are shown in figure 3. Here it can be appreciated that all the resulting RMSEs are below $1 \mathrm{~Hz}$. We notice that in this case the number of non-equivalent dihedrals (16) is significantly lower than the number of experimental data (78). This means that data are redundant and the procedure can detect potential inconsistencies between experimental data.

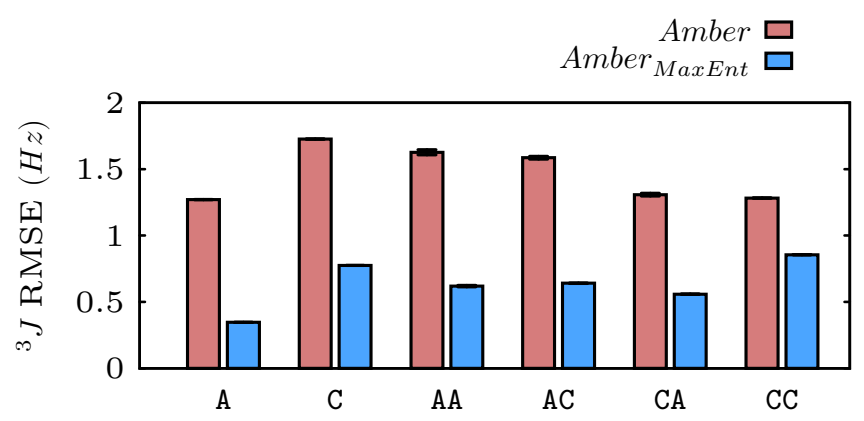

Figure 3: ${ }^{3} J$ RMSE for each system with the Amber force-field and the Amber MaxEnt $_{\text {force-field obtained with }}$ the self consistent refinement.

Back calculated ${ }^{3} J$ couplings for each torsion and Karplus parameters are provided in Supporting Information (table S3,S4,S5). The effect of the corrections on the one-dimensional free-energy profiles associated with all the dihedral angles is shown in Supporting Information (figure S10,S11)

\subsection{Validation on RNA Tetranu- cleotides}

The corrected force field is then validated on two RNA tetranucleotides, AAAA and CCCC. In a previous work ${ }^{17}$ we have shown that on such systems a significant improvement of the agreement with NMR solution experiments can be obtained
Table 2: Lagrangian multipliers associated to each torsional angle used in the self consistent procedure together with the associated Karplus parameters used to back calculate ${ }^{3} J$ scalar couplings. The third column specifies to which system the corrections have to be applied. Karplus relations used are in the form ${ }^{3} J(\theta)=$ $A \cos ^{2}(\theta+\varphi)+B \cos (\theta+\varphi)+C \sin (\theta+\varphi) \cos (\theta+$ $\varphi)+D . \chi^{\prime}$ is defined as $H 1^{\prime}-C 1^{\prime}-N 1 / N 9-C 6 / C 8$ along with a phase shift of $60^{\circ}$.

\begin{tabular}{|c|c|c|c|}
\hline Coupling & Torsion $\theta$ & Base & $\begin{array}{c}\text { Lagrangian } \\
\text { multiplier } \\
\left(H z^{-1}\right)\end{array}$ \\
\hline${ }^{3} J_{H 1^{\prime} H 2^{\prime}}$ & $v_{1}$ & $\mathrm{~A}, \mathrm{C}$ & 0.4393 \\
\hline${ }^{3} J_{H 2^{\prime} H 3^{\prime}}$ & $v_{2}$ & $\mathrm{~A}, \mathrm{C}$ & 0.0570 \\
\hline${ }^{3} J_{H 3^{\prime} H 4^{\prime}}$ & $v_{3}$ & $\mathrm{~A}$ & 0.4009 \\
\hline${ }^{3} J_{H 4^{\prime} H 5^{\prime}}$ & $\gamma^{\prime}$ & $\mathrm{A}, \mathrm{C}$ & 0.3316 \\
\hline${ }^{3} J_{H 4^{\prime} H 5^{\prime \prime}}$ & $\gamma^{\prime \prime}$ & $\mathrm{A}, \mathrm{C}$ & -0.2077 \\
\hline${ }^{3} J_{H 3^{\prime} P}$ & $\varepsilon_{1}$ & $\mathrm{~A}, \mathrm{C}$ & -0.2358 \\
\hline${ }^{3} J_{H 5^{\prime} P}$ & $\beta_{2}$ & $\mathrm{~A}, \mathrm{C}$ & -0.0237 \\
\hline${ }^{3} J_{H 5^{\prime \prime} P}$ & $\beta_{2}$ & $\mathrm{~A}, \mathrm{C}$ & -0.0700 \\
\hline${ }^{3} J_{C 2^{\prime} P}$ & $\varepsilon_{1}$ & $\mathrm{~A}, \mathrm{C}$ & 0.2015 \\
\hline${ }^{3} J_{C 4^{\prime} P}$ & $\varepsilon_{1}$ & $\mathrm{~A}, \mathrm{C}$ & 0.2010 \\
\hline${ }^{3} J_{H 1^{\prime} C 4}$ & $\beta_{2}$ & $\mathrm{~A}, \mathrm{C}$ & 0.1923 \\
${ }^{3} J_{H 1^{\prime} C 2}$ & $\chi$ & $\mathrm{A}$ & 0.1758 \\
\hline${ }^{3} J_{H 1^{\prime} C 8}$ & $\chi^{\prime}$ & $\mathrm{C}$ & 0.4270 \\
${ }^{3} J_{H 1^{\prime} \mathrm{C} 6}$ & & $\mathrm{~A}$ & -0.4068 \\
\hline
\end{tabular}


penalizing $\alpha(g+) / \zeta(g+)$ conformations. These conformations are associated to intercalated structures ${ }^{14,17}$ that are incompatible with solution experiments. We call here Amber $_{\alpha \zeta}$ a potential obtained adding to Amber a two dimensional Gaussian potential centered on the $\alpha(g+) / \zeta(g+)$ conformation with a standard deviation of $0.7 \mathrm{rad}$ and height $8 \frac{\mathrm{kJ}}{\mathrm{mol}}$. The Lagrangian multipliers discussed above were obtained as corrections to be applied on the Amber force field. We here perform a new self-consistent fit with identical simulation parameters using as prior distribution the Amber $_{\alpha \zeta}$ potential and call Amber $\alpha \zeta$ MaxEnt the resulting force field. We also define the Amber $_{\alpha \zeta+\text { MaxEnt }}$ force field as the one obtained by adding the corrections obtained in the previous section on top of the Amber $\alpha \zeta$ force-field, without repeating the self-consistent refinement. In order to asses the performance of Amber, $\operatorname{Amber}_{\alpha \zeta}$, Amber $_{\alpha \zeta+\text { MaxEnt }}$ and Amber $\alpha \zeta$ MaxEnt we performed the same analysis as in refs ${ }^{14,17}$ on AAAA and CCCC. This analysis is made by reweighting the trajectories described in ref ${ }^{17}$. For each force field, we evaluate the RMSE associated to scalar coupling as well as the number of violations and false positives in contacts predicted by nuclear Overhauser experiments (NOEs). NOEs are particularly important in tetranucleotides since they are sensitive to intercalated structures erroneously obtained using the Amber force field that have been previously reported ${ }^{14-17}$. We notice that NOEs might not be visible for many reasons others than the distance is too large. This often happens with large RNAs and proteins and can be due to (1) one or both of the involved resonances are broader than others due to local conformational flexibility at an intermediate rate (microsecond to millisecond), or (2) chemical exchange with solvent protons. All the observed signals in these small systems have similar linewidths (i.e. no intermediate conformational exchange) and only non-exchangeable protons are analyzed. Additionally, for a similar tetranucleotide (GACC) it was shown that intercalated structures would lead to peaks that would be easy to detect because they would appear in unique and uncrowded regions of the spectra. ${ }^{57}$ Comparison of MD with NMR for the tetranucleotides is reported in figure 4. As it can be seen, the MaxEnt corrections improve the agreement with experimental scalar couplings for AAAA and CCCC with respect to both Amber and Amber $_{\alpha \zeta}$ force fields. When considering the NOEs, it can be appreciated that the largest improvement with respect to Amber originates from the $\alpha \zeta$ correction, as previously suggested. Interestingly, the MaxEnt corrections further decrease the number of false positives in CCCC and the number of violations in AAAA. We summarize the agreement with experimental NOEs using the NMR score defined in $\operatorname{Ref}^{14}$. When comparing Amber $_{\alpha \zeta \text { MaxEnt }}$ with Amber $\alpha \zeta+$ MaxEnt it can be noticed that performing a new self-consistent fit starting from Amber $_{\alpha \zeta}$ represent a better choice since it improves both the RMSE and the total NMR agreement. We remark that this is a completely independent validation since experimental data for AAAA and CCCC were not considered in the self-consistent force-field refinement procedure. Moreover, we stress that the validation is made on systems that are different from those used in the fitting procedure. This suggests the corrections to be portable to larger RNA molecules. We finally notice that if the magnitude of the correcting potential is larger than a few $k_{B} T$ the reweighting procedure can lead to very poor sampling ${ }^{58,59}$. To assess the confidence in the reweighting we computed both the Kish's effective sample size ${ }^{60}$ and the statistical error on the RMSE. The Kish's effective sample sizes are respectively 10 (CCCC) and 29 (AAAA) for the Amber $\alpha \zeta$ MaxEnt potential, to be compared to 4000 frames in the unbiased trajectories. Despite these numbers might seem low, the impact of the reweighting procedure on the estimated RMSE is better described by its statistical error. Although the statistical error is significantly increased in the reweighted ensemble (see figure 4), its value is still small enough to allow for a proper comparison between the RMSEs. The structural ensembles obtained with Amber, $\operatorname{Amber}_{\alpha} \zeta$ and Amber $\alpha \zeta$ MaxEnt are also shown in figure 5. It can be appreciated that in both AAAA and CCCC the effect of the MaxEnt corrections is to penalize structures with high value of root-mean-square deviation (RMSD) after optimal superposition from the ideal A-form conformation, which are related to wrongly predicted intercalated conformations. 
AAAA

CCCC

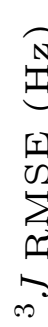

${ }^{3} J$ Coupling
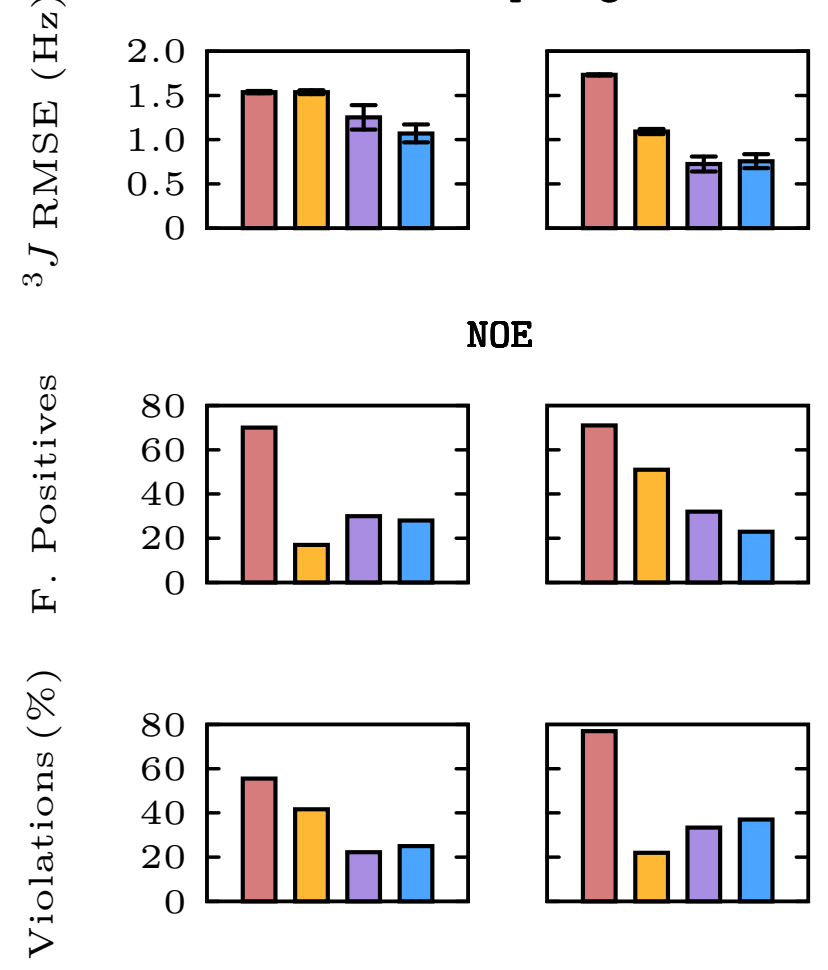

Tot. NMR Agreement

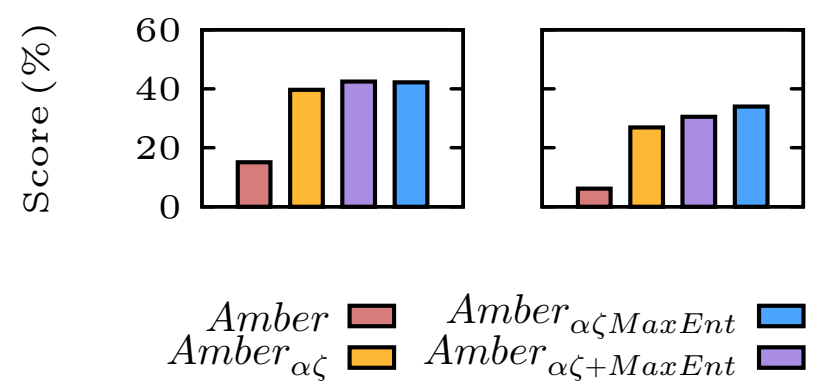

Figure 4: Agreement with the NMR solution experiments for Amber, Amber $\alpha \zeta$ and Amber $\alpha \zeta$ MaxEnt. The number of distance false positives represent the MD predicted NOEs not observed in the experiments.
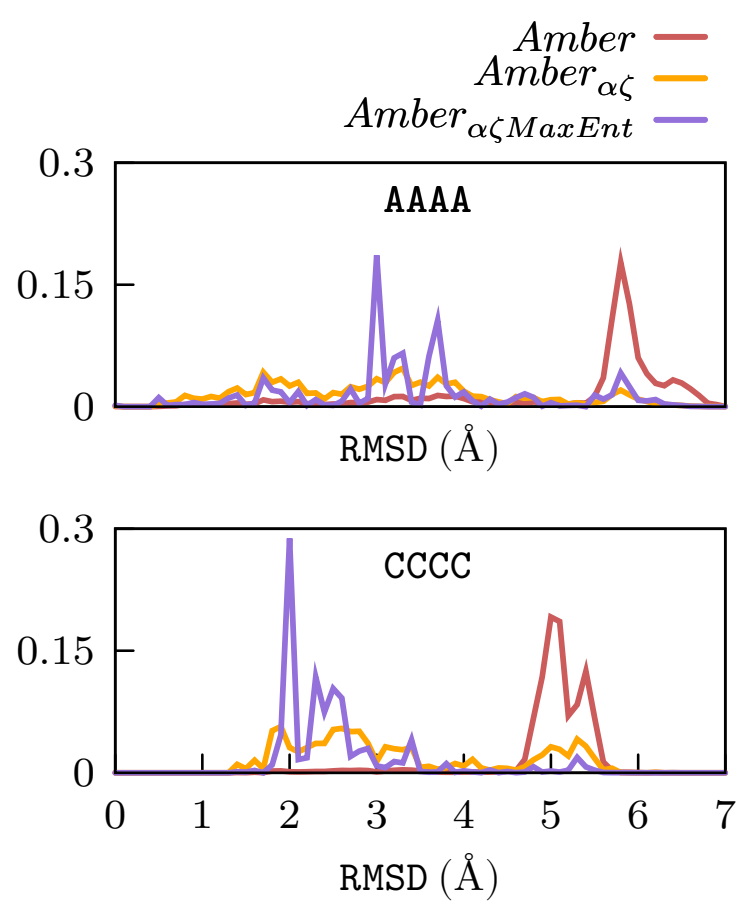

Figure 5: Structural ensembles obtained with Amber, Amber $_{\alpha} \zeta$ and Amber $\alpha \zeta$ MaxEnt . Ensembles are represented by showing the histogram of the RMSD from the ideal A-form conformation. Both $\operatorname{Amber}_{\alpha} \zeta$ and Amber $_{\alpha \zeta \text { MaxEnt }}$ show a significant decrease in the population of the high RMSD structures which are associated to intercalated conformations. 


\section{Discussions}

In this paper we introduced a framework to enforce on the fly noisy data from bulk experiments on molecular dynamics simulations. In the first part (see subsection 1 of Method) we discussed the case of experiments without noisy tolerance. This procedure is completely equivalent to the MaxEnt procedure discussed by Chodera and Pitera ${ }^{26}$ and share many similarities with the experimentally directed simulation (EDS) introduced by White and Voth ${ }^{27}$. In particular, the only difference between the implementation of the MaxEnt procedure used here and EDS is that we here used a different optimization procedure to find the Lagrangian multipliers. In the second subsection of Method we extend the previous approach so as to take into account experimental uncertainties. Several Bayesian approaches have been discussed to model experimental errors in similar contexts (see e.g. ${ }^{29,30,61-63}$ ). Methods have been described to reweight a pre-computed ensemble of structures so as to match experimental averages $29,63,64$. We here apply the MaxEnt procedure on an extended system where fictitious variables are introduced that take into account the discrepancy between theory and experiment. A suitably chosen prior distribution for these variables allows one to control the accuracy of the fitting and to embed in the calculation the confidence in the original force field.

The procedure is iterative and is completely encoded in the update rule stated in Equation 8. It is important to notice that a similar equation could be obtained using theoretical approaches different from the one introduced in this paper. For instance, one could decide to maximize the posterior as a function of the residuals $\varepsilon$ as it is done in $\operatorname{Ref}^{29}$, instead of computing their average value. More comments on this analogy can be found in Supporting Information (section 3).

We notice that other methods have been proposed in the past to model noisy data within the MaxEnt framework. For instance, Chen and Rosenfeld $^{65}$ have proposed to introduce a Gaussian prior on the Lagrangian multipliers. The Laplace prior on the additional variables used here has a similar effect, and allows the range of values for the Lagrangian multipliers to be explicitly controlled.
An alternative formulation of the MaxEnt procedure discussed here can be obtained by replacing the time averages with averages performed on an ensemble of molecular dynamics simulations ${ }^{21,22}$. Replica averaging only converges to MaxEnt when an infinite number of replicas is simulated ${ }^{23,24}$ and implies an intrinsic statistical error in the averages when used with a finite number of replicas. Replica formalism has been extended so as to take into account experimental errors ${ }^{29,30}$. In this context, we preferred to use an iterative procedure since it allows Lagrangian multipliers to be estimated on the fly. The statistical error that in our procedure arises from the finite length of the simulation can be assessed by standard blocking analysis.

The tests that we performed on a model onedimensional system with a bi-modal distribution allow to easily understand the effects of the chosen parameters on the resulting ensembles. In particular, the variance of the prior distribution used for the additional variables can be used to tune the relative weight of the original model and of the enforced experimental data. A Laplace prior for these variables allows for outliers to be tolerated.

We then applied the method to an important open problem, that is the refinement of a force field in order to reproduce available NMR data for RNA oligomers. At first we use our method to enforce all the ${ }^{3} J$ scalar couplings available for the four nucleosides. Since the free-energy landscape of nucleosides have significant barriers, we here combined the approach with an enhancedsampling method based on multiple replicas. This can be straightforwardly done in our formulation since the on-the-fly estimated Lagrangian multipliers can be instantaneously transferred to the biased replicas. The results display a significantly reduced RMSE with respect to experimental data when compared to the original Amber force field. This is expected, since the validation is made against the same dataset used for the training. However, this confirms that the methodology converges to the correct result also in a non trivial model system. We also observe that the employed couplings are unevenly distributed along the RNA backbone. If desired, one could associate a lower value of $\sigma$ to the individual couplings that are considered more relevant so as to increase their weight 
in the fitting procedure.

The method is then applied to the self-consistent force-field fitting for two RNA nucleotides (A and $\mathrm{C}$ ), employing a variety of data measured for several systems ( $\mathrm{A}$ and $\mathrm{C}$ nucleosides, as well as $\mathrm{ApA}, \mathrm{ApC}, \mathrm{CpA}$, and $\mathrm{CpC}$ dinucleoside monophosphates). Also here, the procedure takes implicitly advantage of the on-the-fly transferability of the Lagrangian multipliers. Our approach reminds the spirit behind the restrained ESP charge model ${ }^{66}$, where equivalent atoms are restrained to have equivalent charges. This is translated here in having same correcting potentials on chemically equivalents dihedrals independently of their position in the sequence. Notice that using a selfconsistent procedure where several terms are restrained to be identical effectively reduces the flexibility of the resulting force field and implicitly decreases its capability to match the experimental data. For instance, in the case of a duplicated term in a single simulation (e.g., the $\chi$ angle in an adenine which appears twice in the ApA dinucleoside monophosphate), our approach is only controlling the sum of the two scalar couplings and not their individual values. In our specific application, the number of independent parameters in the force field is 16 , which should be compared with 78 independent experimental data. In this respect, it is important to notice that in this application the calculation of the RMSE, which depends also on the non-explicitly controlled observables, allows for a rigorous cross validation of the method.

The functional form of the corrections derived here, which is proportional to the Karplus equations, is compatible with the one of dihedral potentials. This suggests the use of scalar coupling data as an alternative to quantum chemistry calculations for force-field parametrization or as a refinement tool on top of quantum-chemistry derived torsions. One might be concerned about the fact that corrections developed to match experimental data on small systems are not necessarily portable to larger systems. However, it must be observed that the standard procedure used in the Amber force field is to refine dihedral potentials based on quantum chemistry calculations performed on small fragments, whose typical size is often below the size of the systems considered in this work ${ }^{49,67}$. As a validation, we per- formed a reweighting of previously published trajectories for two RNA tetranucleotides (AAAA and CCCC). In spite of their apparent simplicity these unstructured oligomers are not described properly by any of the current versions of the Amber force field ${ }^{15}$. Our results show that the corrections are portable and significantly improve the description of these tetranucleotides. The resulting RMSEs are below $1 \mathrm{~Hz}$, which is the typical difference between alternate Karplus equations. The development of a force field that consistently describes all nucleotides and dinucleosides, as well as its validation on tetranucleotides and larger systems, is left as a subject for a future investigation.

In conclusion, we introduced a novel procedure that allows experimental errors to be explicitly modeled in a MaxEnt framework. The method is applied to the self-consistent force-field fitting on RNA systems. Results indicate that the obtained force-field corrections are portable and suggest a new paradigm for empirical force-field refinement.

\section{Acknowledgement}

Massimiliano Bonomi, Carlo Camilloni, Michele Parrinello, Omar Valsson, Gregory Voth, and Andrew White are acknowledged for carefully reading the manuscript and providing several useful suggestions. Doug Turner and Scott Kennedy are acknowledged for useful discussions on the interpretation on NOE spectra. The research leading to these results has received funding from the European Research Council under the European Union's Seventh Framework Programme (FP/2007-2013) / ERC Grant Agreement n. 306662, S-RNA-S.

Supporting Information Available: Three sections discussing (1.1) the case of correlated observables, (1.2) the MaxEnt algorithm scheme, (1.3) a family of priors for modelling errors, (1.4) an extension of the method to the case of many restraints with the same error, (1.5) the selfconsistent fit algorithm scheme, (1.6) the RMSD between different Karplus parameters, (2) a one dimensional model system to highlight the features of different error models and (3) a comparison of MaxEnt and maximum a posteriori. Plots of different prior functions (S.1); one dimen- 
sional model system (S.2); RMSE as function of $\sigma$ (S.3); correcting potential on Adenosine $\chi^{\prime}$ torsion (S.4); sample Plumed input file for Adenosine (S.5); sample Plumed input files for the selfconsistent procedure for Adensosine, ApA and Apc (S.6, S.7, S.8, S.9); free-energy profiles in the refined Amber ${ }_{\alpha \zeta \text { MaxEnt }}$ force field for A, C, ApA, $\mathrm{ApC}, \mathrm{CpA}, \mathrm{CpC}$ (S.10, S.11); comparison of maximum entropy and Maximum a posteriori (S.12); ${ }^{3} \mathbf{J}$ scalar couplings and Lagrangian multipliers for A, G, C, U (Tables S.1, S.2); ${ }^{3}$ J scalar couplings and Lagrangian multipliers for A, C, ApA, ApC, $\mathrm{CpA}, \mathrm{CpC}$ in the self consistent procedure (Tables S.3, S.4, S.5); standard deviation between ${ }^{3} \mathrm{~J}$ scalar coupling using various sets of Karplus parameters. This material is available free of charge via the Internet at http://pubs.acs.org/.

\section{References}

(1) Banáš, P.; Hollas, D.; Zgarbová, M.; Jurečka, P.; Orozco, M.; Cheatham, T. E.; Šponer, J.; Otyepka, M. J Chem Theory Comput 2010, 6, 3836-3849.

(2) Colizzi, F.; Bussi, G. J Am Chem Soc 2012, 134, 5173-5179.

(3) Yildirim, I.; Park, H.; Disney, M. D.; Schatz, G. C. J Am Chem Soc 2013, 135, 3528-3538.

(4) Di Palma, F.; Colizzi, F.; Bussi, G. RNA 2013, 19, 1517-1524.

(5) Musiani, F.; Rossetti, G.; Capece, L.; Gerger, T. M.; Micheletti, C.; Varani, G.; Carloni, P. J Am Chem Soc 2014, 136, 15631-15637.

(6) Heldenbrand, H.; Janowski, P. A.; Giambaşu, G.; Giese, T. J.; Wedekind, J. E.; York, D. M. J Am Chem Soc 2014, 136, 7789-7792.

(7) Šponer, J.; Banáš, P.; Jurečka, P.; Zgarbová, M.; Kührová, P.; Havrila, M.; Krepl, M.; Stadlbauer, P.; Otyepka, M. J Phys Chem Lett 2014, 5, 1771-1782.

(8) Giambaşu, G. M.; York, D. M.; Case, D. A. RNA 2015, 21, 963-974.
(9) Pinamonti, G.; Bottaro, S.; Micheletti, C.; Bussi, G. Nucleic Acids Res 2015, 43, 72607269.

(10) Chen, A. A.; Garcia, A. E. Proc Natl Acad Sci U S A 2013, 110, 16820-16825.

(11) Kührová, P.; Banáš, P.; Best, R. B.; Šponer, J.; Otyepka, M. J Chem Theory Comput 2013, 9, 2115-2125.

(12) Miner, J. C.; Chen, A. A.; García, A. E. Proc. Natl. Acad. Sci. U. S. A. 2016, 113, 66656670.

(13) Kuhrová, P.; Best, R. B.; Bottaro, S.; Bussi, G.; Šponer, J.; Otyepka, M.; Banáš, P. J Chem Theory Comput 2016, 12, 45344548.

(14) Condon, D. E.; Kennedy, S. D.; Mort, B. C.; Kierzek, R.; Yildirim, I.; Turner, D. H. J Chem Theory Comput 2015, 11, 2729-2742.

(15) Bergonzo, C.; Henriksen, N. M.; Roe, D. R.; Cheatham, T. E. RNA 2015, 21, 1578-1590.

(16) Bottaro, S.; Gil-Ley, A.; Bussi, G. Nucleic Acids Res 2016, 44, 5883-5891.

(17) Gil-Ley, A.; Bottaro, S.; Bussi, G. J Chem Theory Comput 2016, 12, 2790-2798.

(18) Bottaro, S.; Banáš, P.; Šponer, J.; Bussi, G. J. Phys. Chem. Lett. 2016, 7, 4032-4038.

(19) Torda, A. E.; Scheek, R. M.; van Gunsteren, W. F. Chem Phys Lett 1989, 157, 289294.

(20) Torda, A. E.; Brunne, R. M.; Huber, T.; Kessler, H.; van Gunsteren, W. F. J Biomol NMR 1993, 3, 55-66.

(21) Best, R. B.; Vendruscolo, M. J Am Chem Soc 2004, 126, 8090-8091.

(22) Lindorff-Larsen, K.; Best, R. B.; DePristo, M. A.; Dobson, C. M.; Vendruscolo, M. Nature 2005, 433, 128-132.

(23) Cavalli, A.; Camilloni, C.; Vendruscolo, M. J Chem Phys 2013, 138, 094112. 
(24) Roux, B.; Weare, J. J Chem Phys 2013, 138, 084107.

(25) Jaynes, E. Proc IEEE 1982, 70, 939-952.

(26) Pitera, J. W.; Chodera, J. D. J Chem Theory Comput 2012, 8, 3445-3451.

(27) White, A. D.; Voth, G. A. J Chem Theory Comput 2014, 10, 3023-3030.

(28) Boomsma, W.; Ferkinghoff-Borg, J.; Lindorff-Larsen, K. PLoS Comput. Biol. 2014, 10, e1003406.

(29) Hummer, G.; Köfinger, J. J Chem Phys 2015, 143,243150 .

(30) Bonomi, M.; Camilloni, C.; Cavalli, A.; Vendruscolo, M. Sci Adv 2016, 2, e1501177e1501177.

(31) Jaynes, E. In The Maximum Entropy Formalism; Levine, R. D., Tribus, M., Eds.; The MIT Press, Cambridge, MA, 1979; pp 1518.

(32) Kullback, S.; Leibler, R. A. Ann. Math. Statist. 1951, 22, 79-86.

(33) Karplus, M. J Am Chem Soc 1963, 85, 28702871

(34) Dannenhoffer-Lafage, T.; White, A. D.; Voth, G. A. J Chem Theory Comput 2016, $12,2144-2153$.

(35) Mead, L. R.; Papanicolaou, N. J Math Phys 1984, 25, 2404-2417.

(36) Darken, C.; Moody, J. NIPs 1991, 1009_ 1016.

(37) Barducci, A.; Bussi, G.; Parrinello, M. Phys Rev Lett 2008, 100, 020603.

(38) Valsson, O.; Parrinello, M. Phys Rev Lett 2014, 113, 090601.

(39) Ferrarotti, M. J.; Bottaro, S.; Pérez-Villa, A.; Bussi, G. J Chem Theory Comput 2015, 11, 139-146.
(40) Lee, C.-H.; Ezra, F. S.; Kondo, N. S.; Sarma, R. H.; Danyluk, S. S. Biochemistry (Mosc) 1976, 15, 3627-3639.

(41) Ezra, F. S.; Lee, C.-H.; Kondo, N. S.; Danyluk, S. S.; Sarma, R. H. Biochemistry (Mosc ) 1977, 16, 1977-1987.

(42) Vokáčová, Z.; Buděšinský, M.; Rosenberg, I.; Schneider, B.; Šponer, J.; Sychrovský, V. J Phys Chem B 2009, 113, 1182-1191.

(43) Pronk, S.; Pall, S.; Schulz, R.; Larsson, P.; Bjelkmar, P.; Apostolov, R.; Shirts, M. R.; Smith, J. C.; Kasson, P. M.; van der Spoel, D.; Hess, B.; Lindahl, E. Bioinformatics 2013, 29, 845-854.

(44) Tribello, G. A.; Bonomi, M.; Branduardi, D.; Camilloni, C.; Bussi, G. Comput Phys Commun 2014, 185, 604-613.

(45) Jorgensen, W. L.; Chandrasekhar, J.; Madura, J. D.; Impey, R. W.; Klein, M. L. J Chem Phys 1983, 79, 926.

(46) Cornell, W. D.; Cieplak, P.; Bayly, C. I.; Gould, I. R.; Merz, K. M.; Ferguson, D. M.; Spellmeyer, D. C.; Fox, T.; Caldwell, J. W.; Kollman, P. A. J Am Chem Soc 1995, 117, 5179-5197.

(47) Pérez, A.; Marchán, I.; Svozil, D.; Sponer, J.; Cheatham, T. E.; Laughton, C. A.; Orozco, M. Biophys J 2007, 92, 38173829.

(48) Joung, I. S.; Cheatham, T. E. J Phys Chem B 2008, 112, 9020-9041.

(49) Zgarbová, M.; Otyepka, M.; Šponer, J.; Mládek, A.; Banáš, P.; Cheatham, T. E.; Jurečka, P. J Chem Theory Comput 2011, 7, 2886-2902.

(50) Hess, B.; Bekker, H.; Berendsen, H. J. C.; Fraaije, J. G. E. M. J Comput Chem 1997, $18,1463-1472$.

(51) Darden, T.; York, D.; Pedersen, L. J Chem Phys 1993, 98, 10089-10092. 
(52) Parrinello, M.; Rahman, A. Phys Rev Lett 1980, 45, 1196-1199.

(53) Bussi, G.; Donadio, D.; Parrinello, M. J Chem Phys 2007, 126, 014101.

(54) Gil-Ley, A.; Bussi, G. J Chem Theory Comput 2015, 11, 1077-1085.

(55) Huang, M.; Giese, T. J.; Lee, T.-S.; York, D. M. J Chem Theory Comput 2014, $10,1538-1545$.

(56) Ancian, B. Annual Reports on NMR Spectroscopy; Elsevier BV, 2010; pp 39-143.

(57) Yildirim, I.; Stern, H. A.; Tubbs, J. D.; Kennedy, S. D.; Turner, D. H. J Phys Chem B 2011, 115, 9261-9270.

(58) Shen, T.; Hamelberg, D. J Chem Phys 2008 , $129,034103$.

(59) Ceriotti, M.; Brain, G. A.; Riordan, O.; Manolopoulos, D. E. Proc. R. Soc. A 2012, 468, 2-17.

(60) Gray, P. G.; Kish, L. Journal of the Royal Statistical Society. Series A (General) 1969, 132, 272.

(61) Rieping, W. Science 2005, 309, 303-306.

(62) Olsson, S.; Frellsen, J.; Boomsma, W.; Mardia, K. V.; Hamelryck, T. PLoS ONE 2013, 8, e79439.

(63) Brookes, D. H.; Head-Gordon, T. J Am Chem Soc 2016, 138, 4530-4538.

(64) Beauchamp, K. A.; Pande, V. S.; Das, R. Biophys J 2014, 106, 1381-1390.

(65) Chen, S. F.; Rosenfeld, R. A Gaussian prior for smoothing maximum entropy models; 1999.

(66) Bayly, C. I.; Cieplak, P.; Cornell, W.; Kollman, P. A. J Phys Chem 1993, 97, 1026910280.
(67) Ivani, I.; Dans, P. D.; Noy, A.; Pérez, A.; Faustino, I.; Hospital, A.; Walther, J.; Andrio, P.; Goñi, R.; Balaceanu, A.; Portella, G.; Battistini, F.; Gelpí, J. L.; González, C.; Vendruscolo, M.; Laughton, C. A.; Harris, S. A.; Case, D. A.; Orozco, M. Nat Methods 2015, 13, 55-58. 


\section{Graphical TOC Entry}

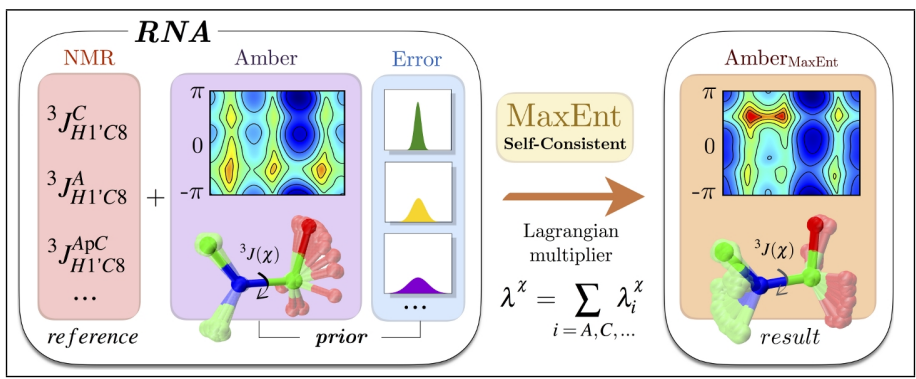

